\title{
First observation of bald patches in a filament channel and at a barb endpoint
}

\author{
A. López Ariste ${ }^{1}$, G. Aulanier ${ }^{2}$, B. Schmieder ${ }^{2}$, and A. Sainz Dalda ${ }^{3,4}$ \\ 1 THEMIS - CNRS UPS 853, C/ vía Láctea s/n, 38200 La Laguna, Tenerife, Spain \\ e-mail: arturo@themis.iac.es \\ 2 Observatoire de Paris, LESIA, 92190 Meudon Cedex, France \\ 3 THEMIS, S.L. C/ vía Láctea s/n, 38200 La Laguna, Tenerife, Spain \\ 4 Instituto de Astrofísica de Canarias, C/ vía Láctea s/n, 38200 La Laguna, Tenerife, Spain
}

Received 27 January 2006 / Accepted 15 May 2006

ABSTRACT

\begin{abstract}
Context. The 3D magnetic field topology of solar filaments/prominences is strongly debated, because it is not directly measureable in the corona. Among various prominence models, several are consistent with many observations, but their related topologies are very different.

Aims. We conduct observations to address this paradigm. We measure the photospheric vector magnetic field in several small flux concentrations surrounding a filament observed far from disc center. Our objective is to test for the presence/absence of magnetic dips around/below the filament body/barb, which is a strong constraint on prominence models, and that is still untested by observations. Methods. Our observations are performed with the THEMIS/MTR instrument. The four Stokes parameters are extracted, from which the vector magnetic fields are calculated using a PCA inversion. The resulting vector fields are then deprojected onto the photospheric plane. The $180^{\circ}$ ambiguity is then solved by selecting the only solution that matches filament chirality rules. Considering the weakness of the resulting magnetic fields, a careful analysis of the inversion procedure and its error bars was performed, to avoid over-interpretation of noisy or ambiguous Stokes profiles. Thanks to the simultaneous multi-wavelength THEMIS observations, the vector field maps are coaligned with the $\mathrm{H} \alpha$ image of the filament.

Results. By definition, photospheric dips are identifiable where the horizontal component of the magnetic field points from a negative toward a positive polarity. Among six bipolar regions analyzed in the filament channel, four at least display photospheric magnetic dips, i.e. bald patches. For barbs, the topology of the endpoint is that of a bald patch located next to a parasitic polarity, not of an arcade pointing within the polarity.

Conclusions. The observed magnetic field topology in the photosphere tends to support models of prominence based on magnetic dips located within weakly twisted flux tubes. Their underlying and lateral extensions form photospheric dips both within the channel and below barbs.
\end{abstract}

Key words. polarization - methods: data analysis - Sun: filaments - Sun: magnetic fields - Sun: photosphere

\section{Introduction}

Since solar filaments/prominences were observed on the solar disc and above the limb, an intense debate has raged the literature on the nature of the physical process that allows cool and dense material to be maintained against gravity in the hot and diluted coronal medium.

Two possible mechanisms have been put forward, fundamentally different and resulting in opposite predictions for the magnetic field topology of prominences. First, the dynamic nature of prominence condensations (as observed by Schmieder et al. 1991; Zirker et al. 1998; Lin et al. 2003, 2005) could arise from a continuous recycling thanks to chromospheric injection along the feet, which typically link prominence bodies to the photosphere (Martin et al. 1994). This injection has been modeled in arcade-like field lines, by a direct driving (Wu et al. 1990), by reconnection-driven flows (e.g. Priest 1998; Litvinenko \& Martin 1999) or by thermal non-equilibrium (e.g. Poland \& Mariska 1986; Karpen et al. 2001). These models have several implications for the topology of prominences, and their associated photospheric channel:

1. both the prominence body and the barbs are formed in nearly flat magnetic arcades;

2. feet and barbs are the footpoints of these arcades;

3. barbs reach the photosphere in filament channels, in a nearly vertical way;

4. the footpoints are anchored in the middle of parasitic polarities;

5. since a barb is made of arcades, the photospheric magnetic field at its endpoint is normal with respect to the small-scale bipole which has the associated parasitic polarity;

6. due to the overall arcade topology, any distant fibril in the filament channel is spicule-like, i.e. oblique or vertical.

Otherwise, the dynamic nature of the condensations could be regarded as of secondary importance if one considers that most condensations remain in near-equilibrium, thus implying the presence of a magnetic support in locally upward-curved 


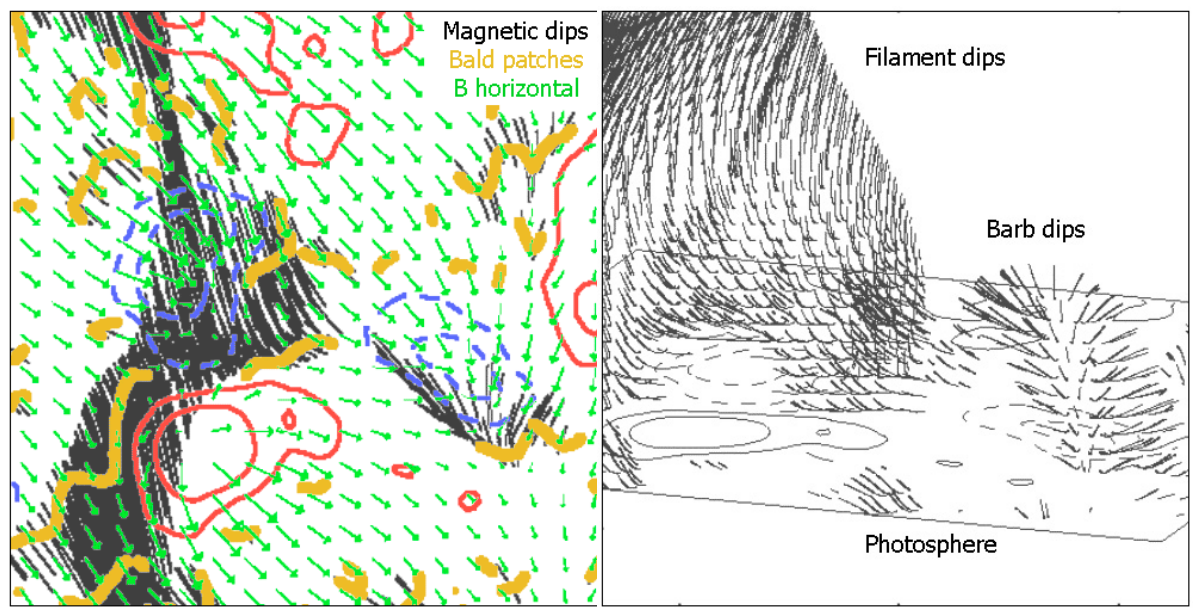

Fig. 1. Modeled magnetic field topology of a filament barb and of the nearby filament channel (adapted from Aulanier et al. 1999), viewed from above (left panel) and in projection (right panel). The blue-dashed (resp. red-continuous) lines stand for contours of $B_{z}$ (photosphere) < 0 (resp. $>0$ ). The green arrows show the photospheric horizontal field. The thick-yellow curves show bald patches (i.e. photospheric magnetic dips) in which, by definition, the horizontal fields go from the $B_{z}<0$ toward $B_{z}>0$ polarities. field lines, i.e. magnetic dips (e.g. Kippenhahn \& Schlüter 1957; Kuperus \& Raadu 1974; Karpen et al. 2003; Karpen 2005). Considering typical observational results from line-ofsight magnetic field measurements in the photosphere (e.g. Martres et al. 1966; Rompolt 1990; Maksimov \& Prokopiev 1995) and within prominences (e.g. Leroy 1977, 1978; Bommier et al. 1986; Leroy 1989; Bommier et al. 1994; Casini et al. 2003; López Ariste et al. 2005), several generic 3D magnetic models have been put forward in this frame. They can be separated into three main classes according to their large-scale topology: quadrupolar fields with dips above a null point (e.g. Malherbe \& Priest 1983; Litvinenko \& Wheatland 2005), sheared and dipped arcades (e.g. Antiochos et al. 1994; DeVore \& Antiochos 2000; DeVore et al. 2005; Aulanier et al. 2002) and homogeneously twisted flux ropes with dips in their windings (e.g. Priest 1990; van Ballegooijen \& Martens 1989; Amari et al. 1999).

Following many specific observational constraints, in particular on the morphology of $\mathrm{H} \alpha$ filament fine-structures and of their lateral extensions (named feet or barbs) and on the chirality rules that define the global magnetic field orientation (as reported in the detailed observational analyses of Martin et al. 1994; Filippov 1995; Martin 1998), a new generation of 3D models have been put forward by Aulaunier \& Démoulin (1998, 2003). They have directly been applied to $\mathrm{H} \alpha$ observations (Aulanier et al. 1999, 2000; Aulanier \& Schmieder 2002, the latter having also been applied to EUV data). These models have been further developed using non-linear (instead of linear) force-free fields, calculated either from extrapolations (Régnier \& Amari 2004), from magneto-frictional methods (Mackay et al. 2000; van Ballegooijen 2004) or from MHD simulations (Lionello et al. 2002). Even though these latest developments considered more realistic and self-consistent physics, and even though they were performed by various independant groups, they fully confirmed the earlier results and predictions:

1. the body of filaments is made of shallow dips within a weakly twisted flux tube that shares many common properties with differentially sheared arcades;

2. the underlying and lateral feet of filaments (i.e. barbs) are also made of magnetic dips, suspended above the photosphere;

3. the continuous distribution of dips in barbs, combined with some projection effects, give the illusion that barbs are made of vertical magnetic fields that connect the filament body to the photosphere;

4. the feet/barb endpoints reach the photosphere in so-called bald patches, where the field is horizontal, on secondary inversion lines located on the side of parasitic polarities, associated with small bipoles in the broard filament channel;

5. the magnetic field orientation in the bald patches is also inverse, not only with respect to the large-scale bipolar field, but also to the small scale bipole;

6. many other low-lying dips and bald patches can be present in filament channels, also being related to parasitic polarities. They can be associated with dark $\mathrm{H} \alpha$ elongated fibrils and with broad EUV extensions.

These predictions are illustrated in Fig. 1, which focuses on a filament barb modeled in Aulanier et al. (1999).

Addressing this paradigm by testing the opposing predictions of both models with new observations has only recently begun. To our knowledge, detailed $\mathrm{H} \alpha$ and EUV spectro-imagery of filaments have been compared with line-of-sight photospheric magnetograms and wide-band photospheric imagery only, both showing magnetic polarities in filament channels, as flux concentrations and inter-granular bright points respectively. So far, the results are inconclusive for the predictions $(2,3,4)$ listed above: Wang (2001) and Lin et al. (2005) found evidence in favor of the arcade models, while on the contrary Zong et al. (2003), Chae et al. (2005) and Schmieder (2005) supported the dip and bald patch models. At this stage, it is clear that measurements of the three components of the photospheric magnetic field vector in filament channels were required, especially in the vicinity of filament barbs, where the predictions of both models differ the most. This is the objective of this work.

THEMIS, in its MTR mode, has been proved to provide vector polarimetry at levels of $10^{-3}$ the continuum intensity for single exposures (López Ariste et al. 2000; Bommier et al. 2005). The detected signals would result in noise levels of $1-2 \mathrm{Mx} / \mathrm{cm}^{2}$ (or Gauss as incorrectly quoted too often in the literature) for the longitudinal flux if the data was to be interpreted as a simple magnetograph. But the seeked tests require vector fields and therefore inversion codes are necessary to infer the field vector, including its inherent distribution inside each pixel. The use of inversion codes with data showing weak magnetic signals poses a problem regarding the reliability of the solution. In this paper we have addressed this problem with a careful analysis of error bars. The inversion technique used (Principal Component Analysis) scans the global parameter space and is therefore able to provide us with true error bars, not affected by the limitations of the approximate formulae often used with least-squares fitting methods. These error bars provide point by point information on the reliability of the different parameters of the vector field, and only those reliable enough are used for the analysis. 


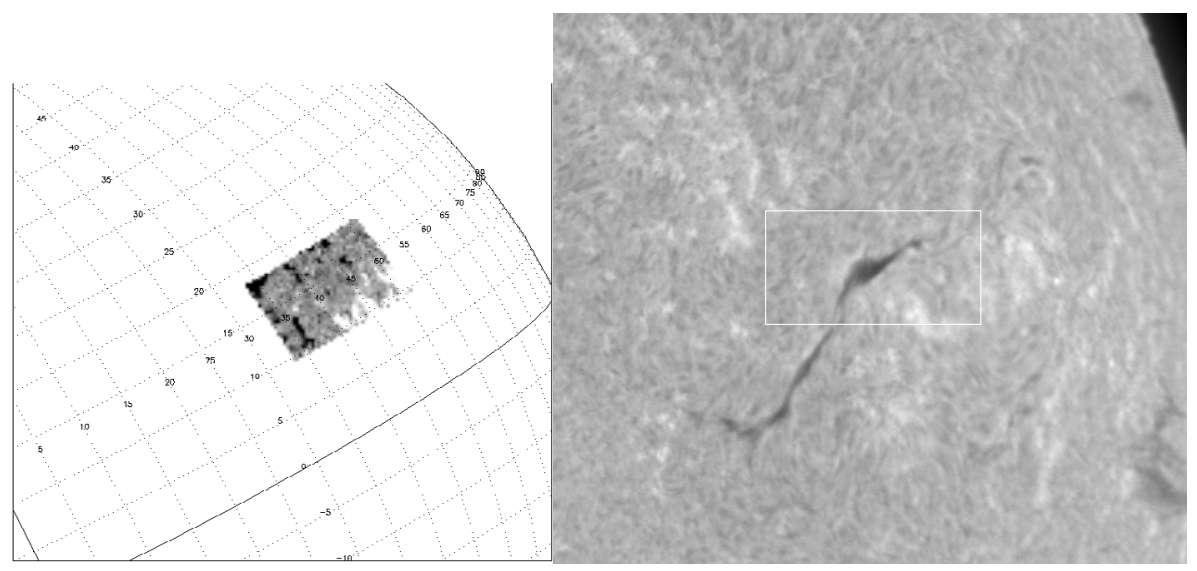

Fig. 2. Left: location of the scan on the solar disc on October 16, 2004. Coordinates are heliocentric longitude and latitude. The embedded image is a magnetogram of the resulting data. Right: corresponding image of the solar disc viewed in $\mathrm{H} \alpha$, observed by BBSO at 16:32 UT, showing the long filament.
This issue of spectropolarimetric accuracy prevents us from deriving trustworthy magnetic fields outside of well-defined flux concentrations, which puts severe constraints on the local regions that can be analyzed in filament channels, which are nearly devoid of vertical flux.

Once the topology of the field has beed determined up to the limits given by error analysis, one problem is still left: the $180^{\circ}$ ambiguity on the azimuth of the field. The different methods available in the literature cannot be trusted in a region with such an absence of dominating magnetic structures as a filament channel. The solution to the ambiguity had to come from a completely different point of view. We used the coherence between the field directions dictated by the chirality rules and one and only one of the two solutions in inclination and azimuth of the field vector made possible from the inversion of the Stokes profiles. This test proved able to solve all the studied cases.

We performed observations of a filament with the THEMIS/MTR instrument, in the frame of the Joint Observing Program (JOP) 178. These new observations, as well as their reduction, are described in Sect. 2. The results are analyzed in Sect. 3 and the resulting magnetic field topologies are given in Sect. 4. The uncertainties and error bars in the magnetic field vector estimations are discussed in Sect. 5. The photospheric topology as derived from observations and including the ambiguity resolution is discussed in the framework of the theoretical paradigm in Sect. 6. The results are summarized in Sect. 7.

\section{Observations and data reduction}

\subsection{THEMIS/MTR observations}

A scan of a quiescent filament roughly centered at 40 degrees of longitude west and $15 \mathrm{deg}$ of latitude north was made on October 16th, 2004. The scan started at 11:20 UT and lasted for more than $2 \mathrm{~h}$ while covering a surface of 240 arcsec in longitude and 84 arcsec in latitude. Figure 2 shows the borders of the scan over an $\mathrm{H} \alpha$ image from BBSO, together with the magnetogram measured with THEMIS embedded in heliographic coordinates. The slit was 1 arcsec wide and oriented along the local solar meridian with the raster movement parallel to the equator. The pixel along the slit was 0.45 arcsec in size and the scanning step accross the slit was 0.8 arcsec in size. We have an anisotropy in spatial resolution smoothed by the average seeing conditions encountered during the scan. For each raster position 6 acquisitions were taken with changing polarimeter configurations to complete a modulation cycle. The modulation was such as to allow the beam-exchange technique (Semel et al. 1993) in the recovery of the Stokes parameters. Three cameras were used in 3 different spectral regions: $\mathrm{H} \alpha$, the region around $630 \mathrm{~nm}$ with the well-known doublet of Fe I lines, and the $\mathrm{Na} \mathrm{D}_{1}$ line region. The results we present here stem from the analysis of the doublet at $630 \mathrm{~nm}$, with the $\mathrm{H} \alpha$ data used for reference only. No analysis has been made of the $\mathrm{Na}_{1}$ data.

Figure 3 shows the $\mathrm{H} \alpha$ map of the region as reconstructed from the red wing of the observed profile, and a magnetogram (saturated to $20 \mathrm{Mx} / \mathrm{cm}^{2}$ ) computed from the Fe I doublet, with heliographic coordinates overplotted. The positive polarity (white on the image) in the southern region of the neutral line is part of a plage region, with strong fields present. The filament channel itself is quite wide, about 1 arcmin in most places, but the filament appears to branch around one of the positive polarity regions towards the south-east. The filament continues out of the region towards the south-east and the branching appears as an over-developed foot.

\subsection{Measurement of photospheric magnetic fields}

The raw data, together with flat field and dark current data, were reduced with SQUV, the new online software provided by THEMIS. The code itself and its performances will be extensively described elsewhere. It computes the usual geometrical and intensity calibrations before applying an optimal demodulation algorithm for each wavelength. The two beams, handled separately up to this point, are then added to increase the signal and diminish atmospheric seeing effects. The level-1 data from SQUV is made of arrays of the four Stokes profiles for each scanned point.

The reduced data corresponding to the Fe I doublet spectral region is fed to an inversion code, also provided by THEMIS as an online tool. The code is a PCA-based algorithm. A MilneEddington atmosphere is considered with thermodynamical parameters ranging to cover the usual possibilities observed in these two lines. The magnetic field, constant with depth, is varied from 0 to $4000 \mathrm{G}$ with all orientations allowed. The two lines of Fe I are synthesized in the same atmosphere, with the only difference of a factor applied to the $\eta_{0}$ parameter (the ratio of line to continuum opacities) corresponding to the ratio of atomic populations of the atomic levels in LTE conditions. The Zeeman effect is solved in simple LS coupling, including anomalous dispersion. A database is made with the Stokes parameters arising from that model using a Monte Carlo algorithm in the manner described by López Ariste \& Casini (2002) to cover the model parameter range. This database is made once and is then read by the inversion code itself each time an inversion is called for. The PCA code is similar to the ones described by Rees et al. (2000), 

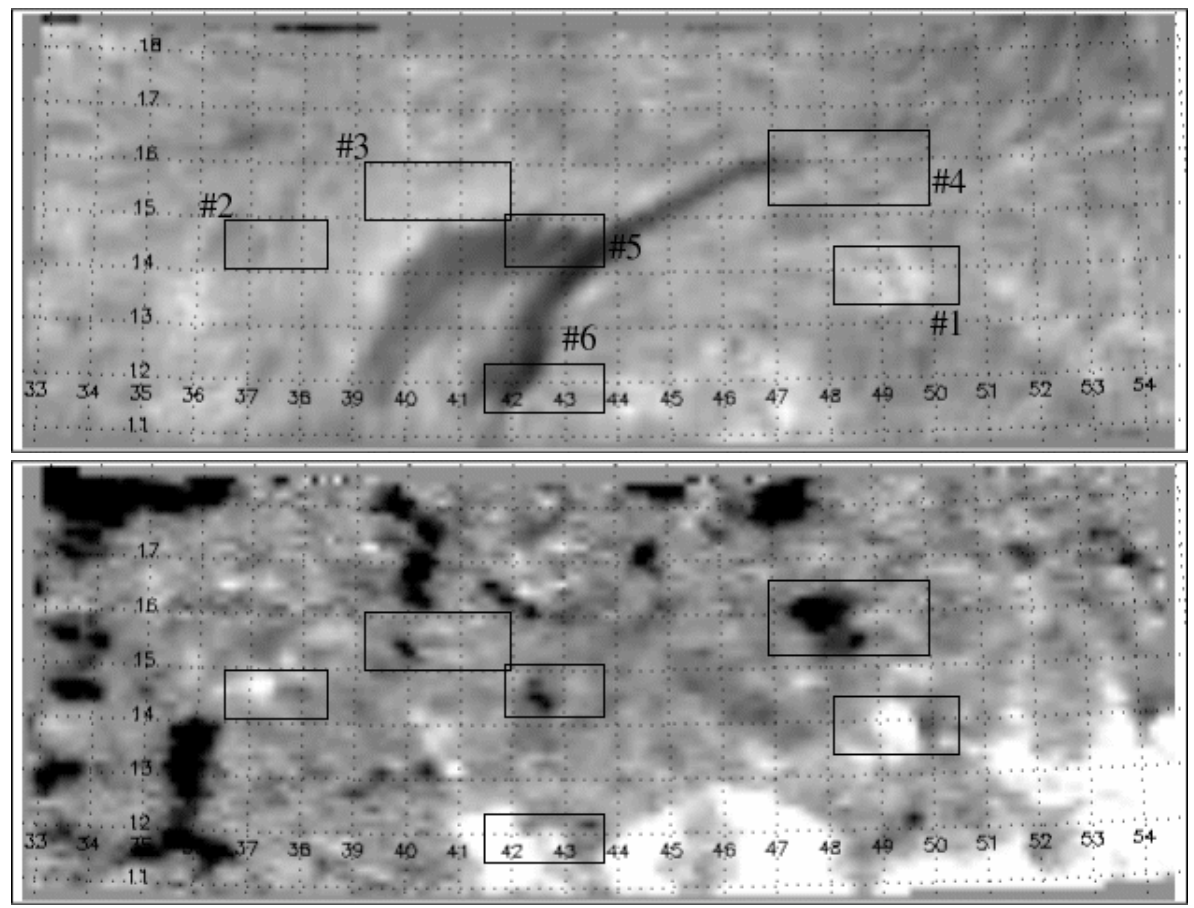

Fig. 3. Reconstructed images of the scan on the red wing of $\mathrm{H} \alpha$ (top, sampled at $130 \mathrm{~m} \AA$ off the line core) and magnetogram (bottom) with overplotted heliographic coordinates. The magnetogram has been saturated to $20 \mathrm{Mx} / \mathrm{cm}^{2}$ for better contrast. Six bipolar structures detected on the magnetogram are outlined and numbered for the study of the magnetic field topology.
Socas-Navarro et al. (2001) and López Ariste \& Casini (2002), and as in those cases it is characterized by its stability and velocity. These two attributes are the reason for its performance as a user-friendly, quasi-real time, inversion code made available to THEMIS observers. Its stability makes it possible that users with no experience in inversion of Stokes parameters can make use of it with no external help and obtain maps of vector magnetic fields of their observed region. Its speed results in those maps being available less than one hour after the scan of a region is ended (for reference, an Intel P3 at $900 \mathrm{MHz}$ performs 50 inversions per second, IO time included).

The output of the inversion code is a series of maps of the scanned region, one per parameter of the Milne-Eddington model atmosphere and a second one for each of the error bars of those parameters. Error bars are computed as follows: for each observed set of Stokes parameters, a Euclidean distance is computed between the PCA coefficients of the observed profiles and each one of the cases present in the database. The minimum distance is given as the solution, but all cases with distances differing by less than $5 \%$ of the minimum distance are also kept. The standard deviation of the model parameters for all those cases is given as the error bar. Since the database in full is considered for this error bar, it lacks many of the problems associated with the computation of errors in the often-used least-squares inversion techniques. All possible ambiguities are included in the calculation of the error bar and in this manner one can estimate how many models would acceptably fit the observations, even if only one among them has been selected as the solution.

\section{Vector magnetic fields in the filament channel}

\subsection{Magnetic maps and bipolar flux concentrations}

The magnetogram at the bottom of Fig. 3 is created from the results of the inversion procedure as $f \cdot B \cdot \cos \gamma$, where $B$ and $\gamma$ are the strength and inclination with respect to the line of sight of the magnetic field, and $f$ is the filling factor, or, in other words, an empiric ratio of the magnetic to the non-magnetic contributions to the final Stokes profiles at each observed solar point. Such a magnetogram is equivalent to what one would get from a magnetograph instrument, except that it has no calibration problems and no saturation effects arising from the use of approximate weak-field formulae, as magnetographs do.

On all figures, the magnetograms have been saturated to $\pm 20 \mathrm{Mx} / \mathrm{cm}^{2}$ (a magnetogram measures magnetic flux density: were the field to be homogeneous throughout the pixel, its strength would be $20 \mathrm{G}$ for a $20 \mathrm{Mx} / \mathrm{cm}^{2}$ flux density) to reveal the magnetic structures in the filament channel. Of course the visible parts of the channel-defining polarities (the positive white in the figure - at the bottom right and the negative - black in the figure - less visible at the top left) become saturated, since their magnetic fluxes are well above this threshold.

Among the many magnetic structures appearing inside the filament channel we notice the presence of many bipolar structures. Such structures have been predicted as inherent parts of the flux rope topology supporting the filament (Aulanier \& Démoulin 1998; Aulanier et al. 1999), and related to particular features of filaments such as their feet/barbs, local interruptions or broadenings (as discussed in Sect. 5). Since the observed region is 40 degrees off disk-center, a first question arises: are these bipolar structures truly solar, or are they just a projection effect due to the angle of the line-of-sight with the local vertical?

\section{2. $180^{\circ}$ amiguity and deprojection}

Since we have performed a full inversion, we have a full vector magnetic field at each point and thus we can attempt a geometric transformation from the reference system defined by the line of sight in which we observe to the reference system defined by the local vertical to which physics is referred.

One difficulty is that spectropolarimetric observations and inversions do not carry information on the orientation, but just on the direction of the transverse field (the projection of the magnetic field vector onto the plane perpendicular to the line of sight, also called the plane of the sky). This is the so-called $180^{\circ}$ ambiguity. Therefore two solutions for the magnetic field are possible differing by field azimuths $180^{\circ}$ apart. 
When performing the trigonometric transformation from the line-of-sight (LOS) reference frame to the local reference frame, the new inclination and azimuth in the local reference frame are computed as functions of both the inclination and azimuth in the LOS reference frame. The ambiguity, translated into the local reference frame, results in two different solutions, for both the local inclination and the local azimuth. A criterion is needed to decide among the two possible solutions.

We disregarded the several algorithms available from the literature for the disambiguation as non-reliable for a region with so many fragmented flux concentrations located in a channel where the coronal prominence field is very far from being potential. So we looked into what chirality rules for filaments had to offer us on this issue.

Looking at the orientations of fine structures in the filament and those of its barbs, observed in $\mathrm{H} \alpha$ with full-disc images, it is clear that the filament is sinistral. As such, the general time-approved rules for the magnetic field in a sinistral filament (Martin 1998), endorse solutions with magnetic fields globally oriented towards the south-east (the bottom left corner of the region in our images). Such a large-scale homogeneous organization of the horizontal fields is justified by the well-known general orientation of chromospheric fibrils in filament channels, which can concern a large area when the surrounding magnetic field is weak (Foukal 1971; Martin 1990).

Since relying exclusively on these rules, we have preferred not to apply them to the full field of view observed by the THEMIS/MTR, but rather to a selection of six small regions, the largest being 30 arcsec long, defined to cover some of the most prominent bipolar structures seen in the magnetogram of Fig. 3. This limitation in our analysis was motivated by the selection of the only bipolar flux concentrations located within the filament channel (i.e. between the plage flux concentrations where the average vertical field is weak), regardless of their position below or beside the filament, in and around which the polarized spectra (in particular the Stokes $V$ profiles) were sufficiently above the noise level so as to ensure that they were neither artefacts of the observational setup nor of the data reduction method.

The 6 regions are labelled in that figure. All the bipoles are in the filament channel which is relevant to the global magnetic configuration; one of them is directly related to a barb. It is to these 6 regions that we apply the chirality rules with the following criteria:

- the inversion code provides solutions for the LOS azimuth limited to the range $0-180$ degrees; thus the inversion results in an implicit continuity of the azimuth for a given region (except for a discontinuty at azimuths of 0 degrees);

- the implicit continuity is deemed physically more acceptable for small regions like the ones selected;

- for the selected regions we conclude therefore that it is appropriate to substitute the 180 degrees ambiguity of each solar point for a region-wide switch of 180 degrees. That is to say that there are only two solutions for each one of the selected regions (and not $2^{N}$ if an ambiguity in a pixel-bypixel basis were considered): either the one provided by the inversor and limited to the 0 to 180 degrees range, or the one resulting from adding 180 degrees to all the points in the region, and thus limited to the 180 through 360 degrees range;

- the two region-wide solutions are governed by the chirality rules.

It is worth noticing that only one of the two possible solutions respected the chirality rules, and this for all and every one of the six regions studied. This was not obvious a priori, since the deprojection of the magnetic field vectors onto the photospheric plane, for a filament located so far from disc center, could have resulted in two solutions that might not have matched the overall chirality rules

\subsection{Comparing longitudinal and vertical fields}

Figures 4-9 show the magnetic field vector in the 6 cases studied after the ambiguity has been resolved in the manner described (Fig. 4 shows both solutions for illustration). Each figure is made of two parts: in the top map the color code traces the longitudinal flux of the region in the LOS reference frame, superposed by the arrows of the transverse field in the local reference frame. Caution should be exercised in this mixing of reference systems. The transverse field represented by the arrows is the magnetic field parallel to the surface of the sun, while the color code is the longitudinal flux in the LOS reference frame, therefore it is not orthogonal to the arrows. The full magnetic field vector in the local reference frame is ploted in the bottom map of each figure (where the arrows represent the transverse field in the local reference frame, as in the top map), where the color code traces the vertical field in that same local reference frame. Thus arrows plus color code in this bottom map reconstruct the full vector magnetic field in the local reference frame.

The reason for these two maps is two-fold. Firstly, as we shall see, the orientation of the arrows will lead us to the main results in the following section, and it was therefore important to place these arrows in a frame (the longitudinal flux) where one could find its spatial connection to the structures discussed throughout the paper. Secondly, the errors in the vertical field (represented as color code in the bottom map of each figure) are considerably larger than those in the orientation of the arrows, thus making the bottom maps more difficult to correctly interpret in physical terms than the top maps, despite being a theoretically better description of the magnetic field vector.

The accrued error bar in the vertical field $B_{z}$ is obvious in the noisy maps of that quantity. Despite the noise, only regions 4 and 6 show a non-clear bipolar structure. For the other 4 regions the bipolar structure seen in the magnetograms is confirmed by $B_{z}$ to be a true solar bipolar structure. Region 1 is perhaps the less convincing of all, with a black polarity region clearly in minority respect to the positive polarity, both in surface coverage and in total flux, which seems definitely uncompensated. This is not unexpected, considering the positioning of region 1 with respect to the filament channel and the plage nature of the positive polarity region limiting that side of the channel. But it is not definitive either.

Regions 4 and 6 show a more random $B_{z}$. The main polarities are still distinguishible. Because of the greater noise in $B_{z}$ it is difficult to be sure of their identification, but the other possibility, a single polarity seen as bipolar because of projection effects, appears as more difficult to sustain.

We therefore conclude that the six regions studied can be safely called bipolar structures as seen in the local reference frame from inspection of the sign of $B_{z}$, the vertical field.

\section{Horizontal fields and magnetic dips}

\subsection{Identification of bald patches}

The horizontal field is shown as oriented arrows in Figs. 4-9. As we shall discuss in the next section, the noise in the orientation of these arrows is smaller than in $B_{z}$ and therefore we are more confident in the value of their orientation. The length of the arrows 

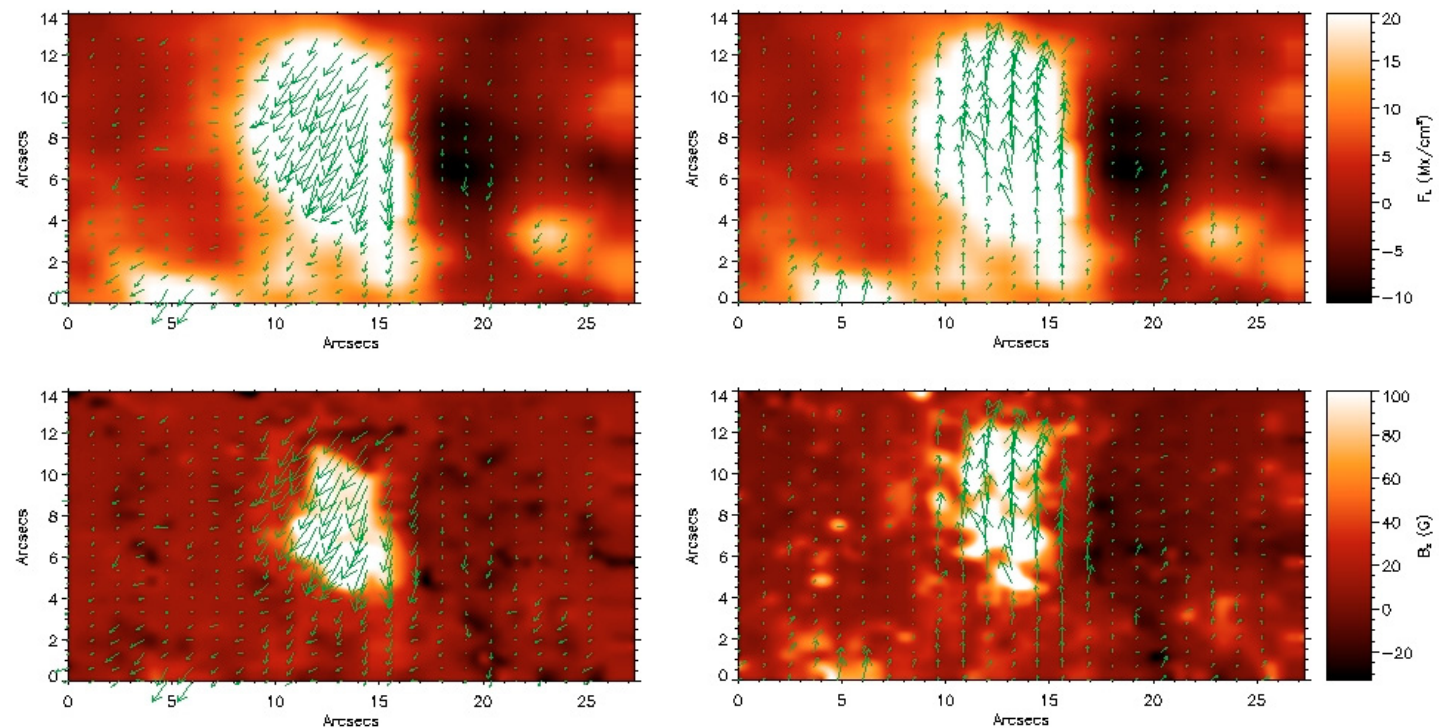

Fig. 4. Vector magnetic field solutions for Case \#1. The arrows on both images represent the projection of the magnetic field onto the plane of the local solar photosphere, that is, the horizontal field. On the top image the color code represents the longitudinal magnetic flux along the line of sight. On the bottom image the color code is the vertical field $B_{z}$, that is the projection of the magnetic field vector onto the local vertical. Note that the full vector can be reconstructed with the arrows and the color code of the bottom image, but that is not the case for the top image. We show for case 1 both solutions for the azimuth ambiguity for comparison. Comparison with chirality rules points to the left solution as the correct one. North is up, and west is right.

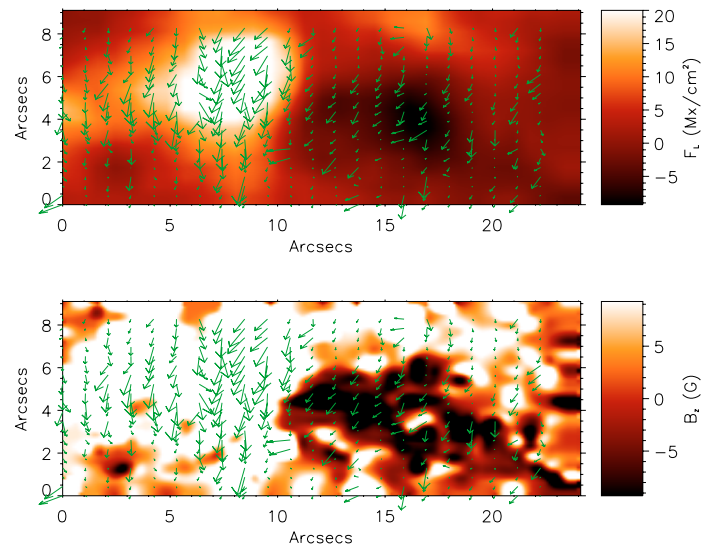

Fig. 5. Vector magnetic field solution for Case \#2. See caption to Fig. 4 for details on the meaning of the arrows and color codes of the top and bottom images. North is up and west is right.

is proportional to the field strength, but it has been saturated to an appropriate threshold for clarity. Since the field strength will neither contribute to our discussion, nor to our conclusions, we shall comment no further on it.

The orientation of the horizontal field reveals the true structure of the field topology at the photosphere. Given a bipolar structure, we get a dip (i.e. a U-loop) on a neutral line separating two flux concentrations of opposite polarities, if the field points from the negative towards the positive polarity. Since we are dealing with photospheric dips, we further refer to them as bald patches (see Titov et al. 1993; Aulanier \& Démoulin 1998). On the other hand an arcade (i.e. an $\Omega$-loop) is seen if the magnetic field points towards the neutral line in the positive polarity and away from it in the negative polarity.

This property readily appears in the equation that defines a bald-patch for $B_{z}=0$ at $z=0$ :

$\boldsymbol{B}_{\mathrm{h}} \cdot \nabla_{\mathrm{h}} B_{z}>0$,
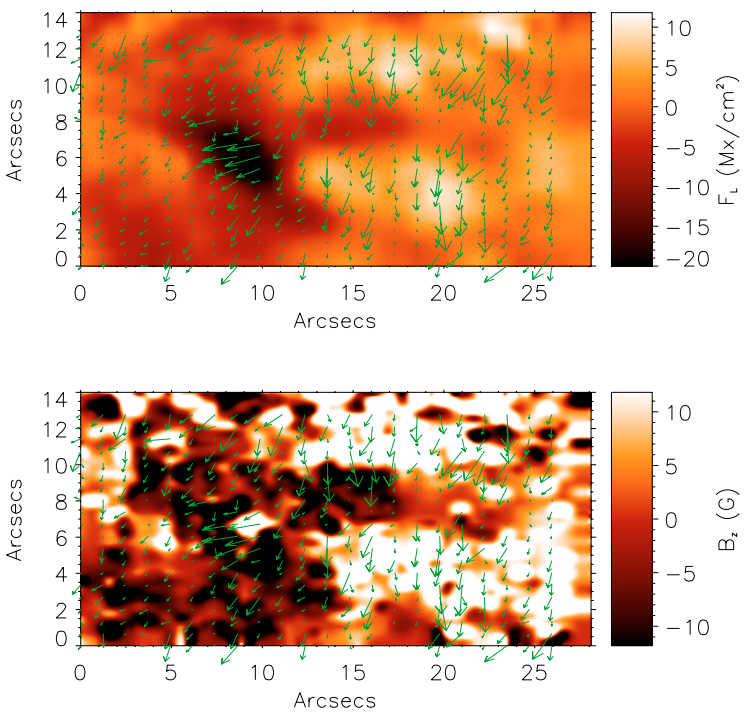

Fig. 6. Vector magnetic field solution for Case \#3. See caption to Fig. 4 for details on the meaning of the arrows and color codes of the top and bottom images. North is up and west is right.

where $\boldsymbol{B}_{\mathrm{h}}=\left(B_{x} ; B_{y}\right)$ is the horizontal field and $\nabla_{\mathrm{h}} B_{z}=$ $\left(\partial B_{z} / \partial x ; \partial B_{z} / \partial y\right)$ is the horizontal gradient of the vertical field. Thus, if $B_{z}$ increases horizontally around $B_{z}=0$ (i.e. passes from a negative to a positive polarity), a bald patch corresponds to a positive horizontal field $B_{\mathrm{h}}$ (i.e. which points in the same direction as the $B_{z}$ gradient, hence toward the positive polarity).

\subsection{The topology of six bipoles in the filament channel}

With these definitions in mind, the six regions are either arcades or bald-patches at the photospheric level.

Provided the negative polarity is truly solar, Case \#1 shows a clear bald patch along the neutral line running north-south, for 

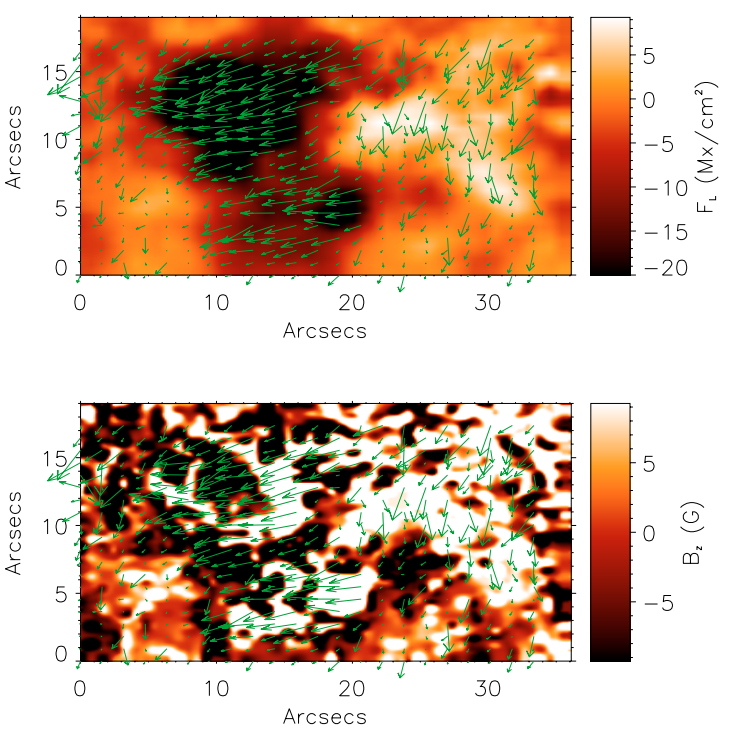

Fig. 7. Vector magnetic field solution for Case \#4. See caption to Fig. 4 for details on the meaning of the arrows and color codes of the top and bottom images. North is up and west is right.

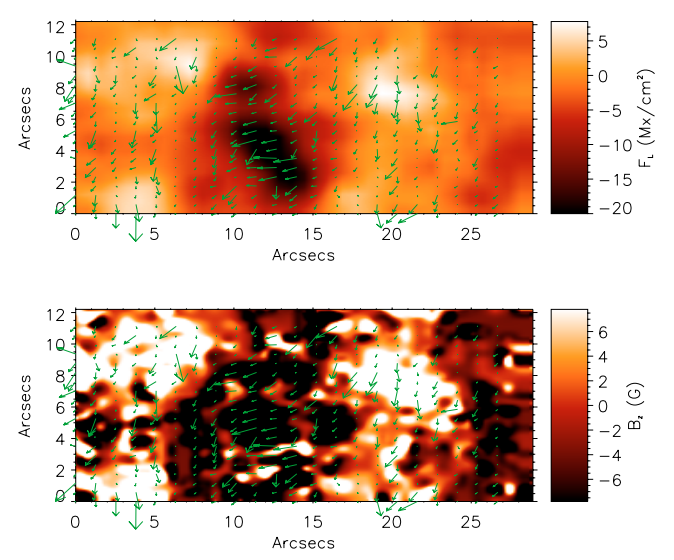

Fig. 8. Vector magnetic field solution for Case \#5. See caption to Fig. 4 for details on the meaning of the arrows and color codes of the top and bottom images. North is up and west is right.

a length of about $5 \mathrm{Mm}$. The field seems to be tilted by an angle of 45 degrees with respect to the local neutral line. It however runs nearly parallel to the large-scale neutral line on top of which the filament body is located. It is weakly of the "inverse polarity" type with respect to the large-scale bipole field, i.e. it points slightly toward the southern positive polarity.

Case \#2 is more difficult to relate either to an arcade or to a bald patch: its horizontal fields appear to run roughly parallel to the local neutral line. There, its orientation is clearly inverse with respect to the large-scale bipole field (hereafter called "globally inverse").

Case \#3, with two positive polarity bands, is more striking: the upper polarity band forms an arcade with the middle negative polarity. But if one looks at a vertical cut at 15 arcsec in the abscissa, and attaches physical significance to the negative polarity band running between the two positive polarity ones (an assumption supported by the strong $B_{z}$ ), what appears is a loopdip structure (already noticed by Pariat et al. 2004, but in the case of an emerging active region) starting in a loop on the upper positive polarity that enters the photosphere in the negative polarity and reappears in the bottom positive polarity. The field orientation above the positive bands is globally inverse.

Case \#4 is complicated because of a noisy $B_{z}$. But it shows a clear arcade in the east-west direction, with field lines roughly orthogonal to the neutral line. There may be a bald patch on the eastern side of the negative polarity, but it is disputable because of the noise in the vertical fields. The field orientation there is globally normal, opposite to inverse. This region is located slightly westward of the northern end of the filament.

In Case \#5, we find again a combination of loops and dips between the three positive polarity patches and the central negative polarity one. The field is clearly globally inverse, since it points southwards. This region is located below a slight northward shift of the filament body, close to the junction between the filament body and barb.

Case \#6 shows two clear bald patches between parasitic negative polarities and the positive polarity towards their south-east, one belonging to the plage. The horizontal field has a globally "normal" polarity inversion. This region is located on the western edge of the filament barb southern endpoint.

\section{Error bars on the model atmosphere parameters}

We now examine the source and magnitude of errors in the parameters, and discuss to what extent our conclusions may be affected by these errors. In practice, it suffices to look at the error bars as provided by the PCA inversion algorithm. Since these error bars have been determined by globally searching in the database for the cases which are the more similar to the observations, we find that all possible errors are translated into the absence of adequate profiles in the database. Such an absence can be seen as a greater-than-usual distance to the solution and therefore a larger number of models lying no further than 5\% of that distance and from which error bars are computed.

Polarimetric sensitivity is placed, for the present telescope setup, at $10^{-3}$ the intensity of the continuum. Systematic errors are expected arising mainly from flat field limitations and from seeing- and slit-induced crosstalk. Flat field limits appear mostly as an I-to-Stokes crosstalk, while both seeing- and slitinduced crosstalks appear as spurious Stokes $V$ signals in all three Stokes $Q, U$ and $V$. Slit-induced crosstalk seems to be negligible in our data thanks to a coalignment estimated to be better than 0.02 arcsec. A few cases of flat field limits are seen throughout the map, more apparent in Stokes $Q$ and $U$, and always at levels near the noise level. The main systematic error in our data appears to be seeing-induced crosstalk. Since it results in the appearance of Stokes $V$-like signals in Stokes $Q$ and $U$ profiles, which cannot be reproduced by any of the models present in the database, its presence results in solution profiles far away from the observed profile and therefore in an accrued number of different models within the 5\% threshold; this translates generally into a larger error bar, as explained in the previous paragraph.

A similar reasoning can be applied to asymmetries in the observed profiles, which can be attributed to gradients with depth of the velocity and magnetic parameters and which are left unexplained in a Milne-Eddington atmospheric model like the one implicit in our solutions. Their appearence in the observed dataset also results in an increased error bar assigned to the point by the inversion algorithm. Obviously all the systematic biases inherent to the inversion algorithm known to-date behave that way too, including the effects of a discrete database.

An example of two characteristic profiles in our data is given in Figs. 10 and 11. It is taken from each of the two main polarities in region 1. The Stokes profiles in Fig. 10 show a strong 

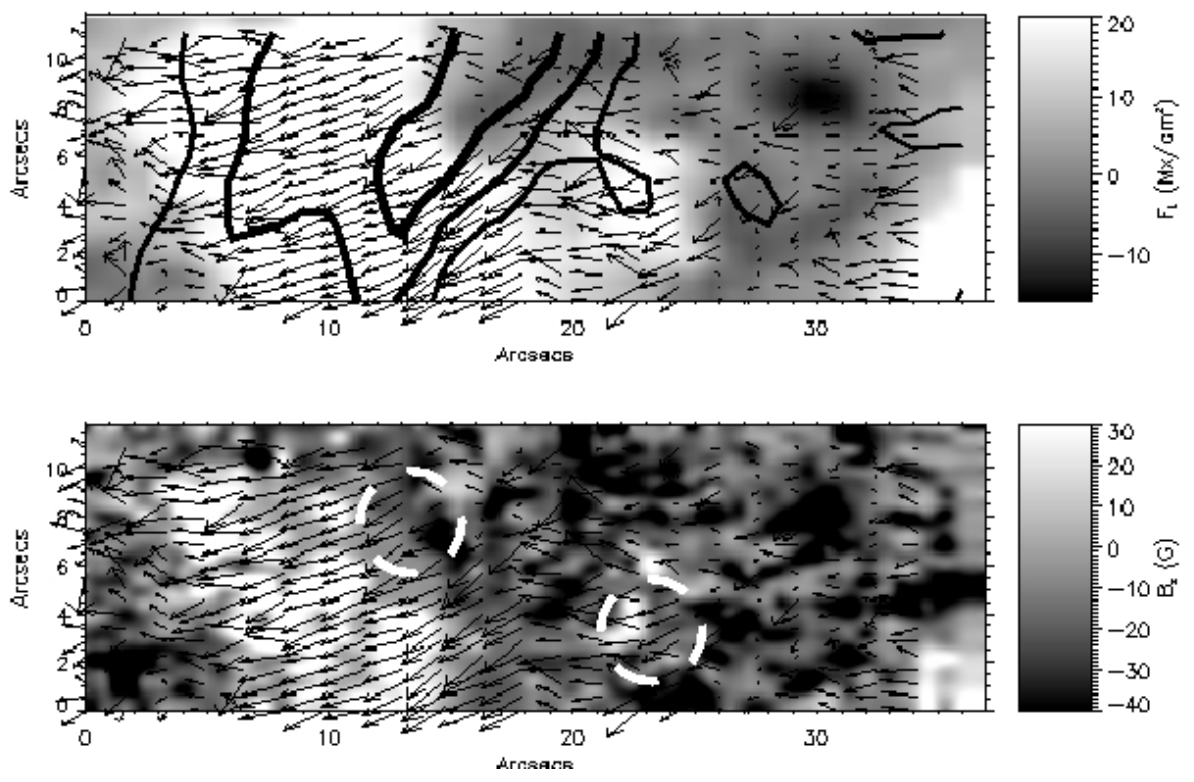

Fig. 9. Vector magnetic field solution for Case \#6. See caption to Fig. 4 for details on the meaning of the arrows and color codes of the top and bottom images. The two brokenline circles on the $B_{z}$ plot mark two clear baldpatches. The continuous lines on the top panel show isocontours of the $\mathrm{H} \alpha$ filament barb; the thin (resp. thick) lines correspond to dark (resp. darker) intensities, due to weak (resp. strong) absorption in $\mathrm{H} \alpha$.
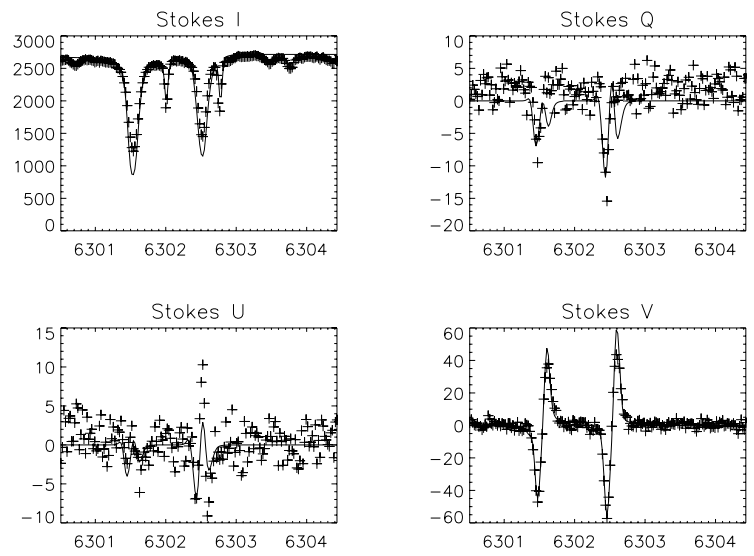

Fig. 10. Stokes profiles, observed (crosses) and inversion fit (continuous line) for a point in the positive polarity of the Case \#1.
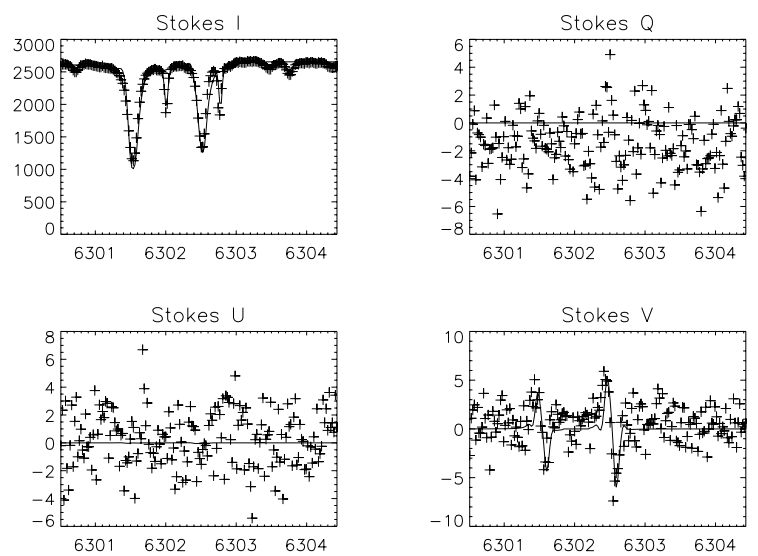

Fig. 11. Stokes profiles, observed (crosses) and inversion fit (continuous line) for a point in the negative polarity of Case \#1.

signal both in linear and circular polarization. The fit given by the solution model is overplotted and reproduces the profiles of both lines, if one excludes the asymmetries, more visible in the $Q$ and $U$ profiles. This is a typical case of strong flux $\left(140 \mathrm{Mx} / \mathrm{cm}^{2}\right)$ for which we can expect a good determination of all the parameters of the atmospheric model, including field
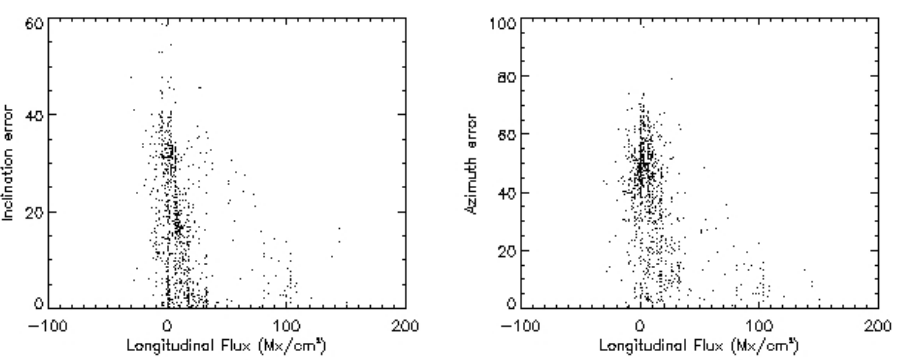

Fig. 12. Scatter plots of error bars as determined by the PCA inversion code for inclination (left) and azimuth (right) of the magnetic field in the LOS reference frame, as a function of longitudinal flux.

azimuth. In Fig. 11 we show an example of one of the smallest signals observed from which some physical information can be still expected to be retrieved. Stokes $Q$ and $U$ are lost in the noise, while Stokes $V$ peaks at roughly 2 or 3 times the noise level. The example corresponds to a longitudinal flux of $8 \mathrm{Mx} / \mathrm{cm}^{2}$, approaching our detection limit for the stated polarimetric sensitivity. At first sight it may appear that no much information can be retrieved from such profiles, but they carry more than what meets the eye: The amplitude of the Stokes $V$ profiles fixes the longitudinal flux, while the ratio for the two lines in the Fe I doublet provides some information on the intrinsic field strength. The absence of Stokes $Q$ and $U$ signals places strong limits on how inclined the field may be (it should be horizontal enough to result in a small $V$ signal, given the inferred field strengths, but not so much as to allow the transverse Zeeman effect to bring $Q$ and $U$ up above the noise level). One can see that even in such a case only the azimuth is completely undetermined. All this back-of-the-envelope reasoning is implicit to the working ways of the inversion algorithm. That can be seen in Fig. 12 where we plot error values as determined by the inversion code for both the LOS inclination (left plot) and the LOS azimuth (right plot) versus longitudinal flux. The plots show that for strong longitudinal fluxes, as in the example shown in Fig. 10, both inclination and azimuth are satisfactorily retrieved, with error bars of the order of 20 degrees at most. For weak longitudinal fluxes, however, the result is almost opposite: while the inclination is still kept in acceptable limits with many cases still producing a 20 to 30 degrees error bar and most of them less than 40 degrees error, the azimuth presents errors 
above 40 degrees in too many cases. This coincides with the picture we developed above from the examination of the profiles in the example of Fig. 11: the inclination is still retrieved in acceptable conditions from most of the cases, but the azimuth is lost.

The change from the LOS to the local reference frame carries a trade in error bars from LOS inclination and LOS azimuth into local inclination and local azimuth. The correspondence is obviously not one-to-one and it depends on the angle between the LOS and the local vertical. For a point at disk center, where LOS and local vertical coincide, inclination is the same in both reference systems. If we move to the limb, though, at 90 degrees between the LOS and the local vertical, LOS azimuth becomes local inclination and viceversa. In general for an angle $\theta$, the error in the local inclination $\delta \vartheta_{B}$ is given by the quadratic addition of the errors introduced by the error in the LOS inclination $\delta \Theta_{B}$ and in the LOS azimuth $\delta \Phi_{B}$, given by the two following expressions:

$\left\|\sin \vartheta_{B}\right\| \delta \vartheta_{B}=\left\|-\cos \theta \sin \Theta_{B}+\sin \theta \cos ^{2} \Theta_{B}\right\| \delta \Theta_{B}$

$\left\|\sin \vartheta_{B}\right\| \delta \vartheta_{B}=\left\|\sin \theta \sin \Theta_{B} \sin \Phi_{B}\right\| \delta \Phi_{B}$,

derived from the use of the cosine theorem in the spherical triangle defined by the local vertical, the LOS and the magnetic field.

The use of the four-parts formula to that same spherical triangle leads to the two expressions which, quadratically added, give the error bar in the local azimuth $\delta \phi_{B}$ as a function again of the errors in the LOS inclination and LOS azimuth:

$$
\begin{aligned}
\left\|\sin \Phi_{B}\left(\cot ^{2} \phi_{B}+1\right)\right\| \delta \phi_{B}= & \left\|\sin \theta\left(\cot ^{2} \Theta_{B}+1\right)\right\| \delta \Theta_{B} \\
\left\|\left(\cot ^{2} \phi_{B}+1\right)\right\| \delta \phi_{B}= & \| \sin \theta \cot \Theta_{B} \frac{\cos \Phi_{B}}{\sin ^{2} \Phi_{B}} \\
& +\cos \theta \sin \Phi_{B} \| \delta \Phi_{B} .
\end{aligned}
$$

The resulting formulae, although complete, are not very intuitive about the dependence of the errors on one or the other parameter. Figure 13 shows the result of these formulae in the form of error bars for the local inclination and local azimuth as a function of the longitudinal flux. The conclusion is that at this angular distance from disk center the switch between azimuths and inclinations is almost completed and therefore the local inclination error mostly depends on the LOS azimuth error and is almost completely undetermined. On the other hand the local azimuth, dependent mostly on the LOS inclination, still produces acceptable error bars. Thus the value of $B_{z}$ is not reliable given the error bars of the local inclination. We can rely, on the other hand, on the orientation of the horizontal field, the local azimuth, seen as the direction of the green arrows in Figs. 4 through 9, and providing us with information on the dip- or arcade-topology of the field.

\section{Comparison with model predictions}

The observed filament has a well developped channel which is nearly free of vertical magnetic fields, except in small dispersed bipoles located at various places with respect to the filament. While the origin of these bipoles is unknown (are they a natural consequence of flux tube emergence as discussed in Aulanier et al. 1999, or are they simply formed by a local subphotospheric dynamo?), their vector magnetic fields are the key to the topology of the filament. This last statement is justified by the orientation of the magnetic fields, which is believed to be homogeneous on the scale of the whole filament channel, so including within the filament itself, and for which local and dynamic
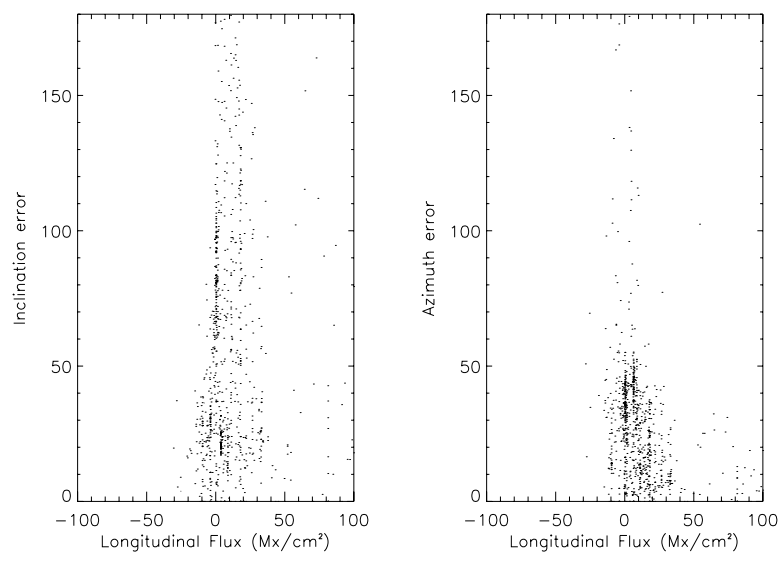

Fig. 13. Scatter plots of error bars for the inclination (left) and azimuth (right) of the magnetic field in the local reference frame, as a function of longitudinal flux.

photospheric flux concentrations must interact, and therefore realign (e.g. through magnetic reconnection) with these large-scale organized overlaying fields. This point is key to relate our measurements to the filament.

Bipoles \#1, 2 and 3 are far from the filament body. All of them possess bald patches and their horizontal fields are of the inverse polarity with respect to the large-scale bipolar field that defines the channel. These properties are consistent with those found with a global weakly twisted flux tube topology for the filament. In this context, the inverse polarity in the photosphere corresponds to the horizontal extension of the flux tube: its lower parts naturally form two so-called "fishbone" structures, one on each side of the neutral line, and between which the configuration is inverse (Filippov 1995). There, the bald patches are a natural consequence of the low altitude perturbation of the flux tube by parasitic polarities: the sheared field lines surrounding the flux tube locally lean toward the parasitic flux concentrations, which result in the formation of low altitude magnetic dips near their footpints, and which can be related to dark elongated fibrils as observed in $\mathrm{H} \alpha$ (Aulanier \& Démoulin 1998; Aulanier et al. 1999) and to broad dark EUV extensions around the filament (Aulanier \& Schmieder 2002). Such photospheric dips, however, are not incorporated in inverse-polarity arcade based models (Martin et al. 1994).

Bipole \#4 is located almost on the main neutral line, a few arcsec away from the northern extremity of the filament as seen in $\mathrm{H} \alpha$. It has a clear arcade topology. It may therefore support, a priori, arcade based models for filament bodies. But the latter models predict that the plasma in filament bodies is continuously and dynamically supplied from chromospheric injection, which implies that a filament arcade should be filled by $\mathrm{H} \alpha$ absorbing material all along its length. This is not the case here. The observations are consistent with a dipped arcade (regardless of its twisted or sheared geometry): the filament material is located at high altitude, within the dipped parts only of the field filament field lines, whose footpoint naturally reaches the photosphere farther away from the 3D distribution of dips (DeVore \& Antiochos 2000; Aulanier et al. 2002; Régnier \& Amari 2004).

Bipole \#5 is placed under the place where the filament body's axis abruptly shifts northward, and where a lateral extension separates from the the body and thus forms a barb. Its topology is hard to infer because the horizontal fields run nearly parallel to the local neutral line, whose precise location and orientation suffer from non-negligible uncertainties (see Sect. 5). Still, the 


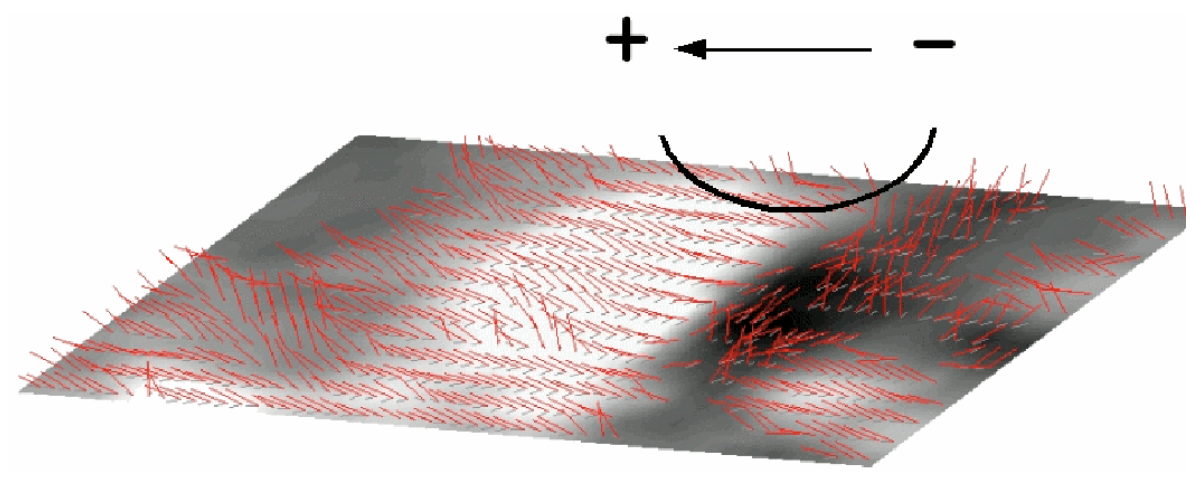

Fig. 14. 3-dimensional respresentation of the vector magnetic field solution for Case \#1. The field vector in the negative/black polarity points towards the neutral line, while in the positive/white polarity points away from it whenever the magnetic field is shaped as a dip, as illustrated in this case. horizontal fields are clearly globally inverse and orthogonal to the filament body. Depending on its topology, it would support either of the filament models (arcades vs. dips). All this region's properties are very similar to those of the largest bipole shown in the center of Fig. 1, adapted from Aulanier et al. (1999), where an underlaying filament foot is present (but not necessarily visible with all projection views since the filament body overlays it): it is in this region that the field is the "most inverse" in the filament model.

The region \#6 shows two bald patch regions (marked by open circles in Fig. 9) with globally normal polarities. The easternmost bipole is placed below the extremity of the barb, which extends a few arsecond further away from the bald patch, on top of the positive dominant polarity region. This is contradictory to arcade based models, that not only claim the barb topology to be that of an arcade, but also claim that barbs point to the middle of parasitic polarities. It is, however, consistent with the predictions of Aulanier et al. (1999): the barbs are formed by a continuous distribution of magnetic dips; the lowermost ones, in the photosphere, have their bottom located on the bald patch, but their horizontal extension (ensured by the vertical filling of the dips by cool plasma) naturally accounts for a shift in the barb endpoint away from the parasitic polarity. The globally normal polarity is not an issue, since the barb extremity can easily be located near the fishbone structure, where the field passes from inverse to normal as one gets away from the filament perpendicularly to the neutral line (as skecthed by Filippov 1995). The barb shown in Fig. 1 is of this type.

\section{Summary}

Using the MTR instrument of the THEMIS telescope, we conducted spectro-polarimetric observations of the northern section of a long filament channel, measuring the four Stokes parameters in the photospheric Fe I doublet at $630 \mathrm{~nm}$ and simultaneously and co-spatially in the $\mathrm{H} \alpha$ line. Our objective was, through complete determination of the photospheric vector magnetic field, to investigate the topology of filament channels that arises from opposite predictions of two different families of models, the first one being based on arcades and the second one on magnetic dips.

The spectro-polarimetric data was reduced using the new code SQUV, especially designed for the MTR, as well as a PCAbased inversion algorithm to infer vector magnetic fields from the Stokes profiles. We focused our attention on several small bipolar flux concentrations located throughout the filament channel and along its lateral extension (i.e. its barb). Thanks to the position of the filament far away from disc center, strong projection effects existed. This led the two solutions for the standard $180^{\circ}$ ambiguity on the magnetic field direction on the plane of the sky to result in fundamentally different vector magnetic fields when projected onto the local solar vertical. Instead of using one algorithm among the many existing ones to solve the $180^{\circ}$ ambiguity, we arbitrarily chose the solution that satisfied the well-known observational chirality rules for filament channel horizontal magnetic field directions, according to the obvious sinistral nature of the filament, as defined by Martin (1998). For each case that we analyzed, only one of the two solutions related with the $180^{\circ}$ ambiguity satisfied these chirality rules.

Our choice for this homogeneous organization of the horizontal fields is justified by the well-known large-scale orientation of chromospheric fibrils in filament channels, as reported e.g. by Foukal (1971) and Martin (1990). This pattern is known not to be present outside of the filament channel, e.g. in the quiet Sun or within plage regions, where the horizontal magnetic fields are believed to be much weaker than the vertical fields (as supported by the observed organization of chromospheric spicules and anemona-shaped groups of fibrils). This special property is then the main one and naturally leads to the presence of bald patches in small bipoles located in filament channels that allow us to associate the topology of the filament channel with that of the filament itself.

The resulting field topologies, filtered by a careful error bar analysis, resulted in a series of conclusions that can be summarized as follows:

- At least four (maybe five) of the six bipoles analyzed in the filament channel have a bald patch topology: this means that both polarities are not connected by an arcade, but rather by a photospheric dip. Figure 14 illustrates the magnetic dip topology in one of the analysed regions, Case \#1. In a dip topology the horizontal field points, by definition, from the negative/black polarity towards the positive/white one, through the neutral line.

- In almost all bald patches, the magnetic fields have an overall inverse polarity with respect to the large scale bipolar field in which the filament channel is embedded.

- The endpoint of the long filament barb corresponds to a small bipole that contains a parasitic polarity and a bald patch (Case \#6).

- It seems that the barb extends further southward from its associated bald patch,

- The northern filament extremity ends slightly eastward of a strong polarity whose topology is that of an arcade.

These measurements clearly support the predictions for dipbased models for prominence barbs as reported in Sect. 1. The third and fourth items reported above clearly show that the barb endpoint is not located within a negative parasitic polarity, but rather a little away from it. This seems to be consistent with the support of cool condensations in magnetic dips within the barb. The last item suggests that the filament body is not made of 
dynamic condensations travelling through an arcade, since otherwise the filament extremity should point at the footpoint of its field line. It is consistent, however, with the geometry of a dipped field line running nearly parallel to the filament axis.

Our results, based on the study of one filament channel, gave observational information on the issue of the long standing paradigm about the topology of the magnetic field in prominence bodies and feet. Some open questions still remain though, mostly due to our choice in the general orientation of the horizontal fields in the filament channel and to our limited statistics over the few studied bipoles. Therefore it is clear that our procedure will have to be repeated in the future for independent verification, hopefully with fine-tuned instrumental setups with ground-based telescops and with future space-borne vector magnetographs.

Acknowledgements. The authors thank the THEMIS staff and all the organizers of the JOP178 observing campaign, in the framework of which this study was performed. THEMIS is a telescope operated by the CNRS (France) and the INAF (Italy) on the Island of Tenerife (Spain). We also thank the referee, who helped us clarify some important points. This work was supported by the European Commission through the RTN programme (European Solar Magnetism Network, contract HPRN-CT-2002-00313).

\section{References}

Amari, T., Luciani, J. F., Mikic, Z., \& Linker, J. 1999, ApJ, 518, L57 Antiochos, S. K., Dahlburg, R. B., \& Klimchuk, J. A. 1994, ApJ, 420, L41 Aulanier, G., \& Démoulin, P. 1998, A\&A, 329, 1125

Aulanier, G., \& Démoulin, P. 2003, A\&A, 402, 769

Aulanier, G., Démoulin, P., Mein, N., et al. 1999, A\&A, 342, 867

Aulanier, G., DeVore, C. R., \& Antiochos, S. K. 2002, ApJ, 567, L97

Aulanier, G., \& Schmieder, B. 2002, A\&A, 386, 1106

Aulanier, G., Srivastava, N., \& Martin, S. F. 2000, ApJ, 543, 447

Bommier, V., Degli'Innocenti, E. L., Leroy, J.-L., \& Sahal-Brechot, S. 1994, Sol. Phys., 154, 231

Bommier, V., Rayrole, J., \& Eff-Darwich, A. 2005, A\&A, 435, 1115

Bommier, V., Sahal-Bréchot, S., \& Leroy, J. L. 1986, A\&A, 156, 79

Casini, R., López Ariste, A., Tomczyk, S., \& Lites, B. W. 2003, ApJ, 598, L67

Chae, J., Moon, Y.-J., \& Park, Y.-D. 2005, ApJ, 626, 574

DeVore, C. R., \& Antiochos, S. K. 2000, ApJ, 539, 954

DeVore, C. R., Antiochos, S. K., \& Aulanier, G. 2005, ApJ, 629, 1122

Filippov, B. P. 1995, A\&A, 303, 242

Foukal, P. 1971, Sol. Phys., 19, 59

Karpen, J. T. 2005, ApJ, in press
Karpen, J. T., Antiochos, S. K., Hohensee, M., Klimchuk, J. A., \& MacNeice, P. J. 2001, ApJ, 553, L85

Karpen, J. T., Antiochos, S. K., Klimchuk, J. A., \& MacNeice, P. J. 2003, ApJ, 593, 1187

Kippenhahn, R., \& Schlüter, A. 1957, Z. Astrophys., 43, 36

Kuperus, M., \& Raadu, M. A. 1974, A\&A, 31, 189

Leroy, J. L. 1977, A\&A, 60, 79

Leroy, J. L. 1978, A\&A, 64, 247

Leroy, J. L. 1989, in Dynamics and Structure of Quiescent Solar Prominences, ASSL, 150, 77

Lin, Y., Engvold, O. r., \& Wiik, J. E. 2003, Sol. Phys., 216, 109

Lin, Y., Wiik, J. E., Engvold, O., Voort, L. R., \& Frank, Z. A. 2005, Sol. Phys., 227, 283

Lionello, R., Mikić, Z., Linker, J. A., \& Amari, T. 2002, ApJ, 581, 718

Litvinenko, Y. E., \& Martin, S. F. 1999, Sol. Phys., 190, 45

Litvinenko, Y. E., \& Wheatland, M. S. 2005, ApJ, 630, 587

López Ariste, A., \& Casini, R. 2002, ApJ, 575, 529

López Ariste, A., Casini, R., Paletou, F., et al. 2005, ApJ, 621, L145

López Ariste, A., Rayrole, J., \& Semel, M. 2000, A\&AS, 142, 137

Mackay, D. H., Gaizauskas, V., \& van Ballegooijen, A. A. 2000, ApJ, 544, 1122

Maksimov, V. P., \& Prokopiev, A. A. 1995, Astron. Nachr., 316, 249

Malherbe, J. M., \& Priest, E. R. 1983, A\&A, 123, 80

Martin, S. F. 1990, in IAU Colloq., 117, ed. V. Ruzdjak, \& E. Tandberg-Hanssen (Springer-Verlag), 1

Martin, S. F. 1998, Sol. Phys., 182, 107

Martin, S. F., Bilimoria, R., \& Tracadas, P. W. 1994, in Solar Surface Magnetism (Dordrecht: Kluwer), 303

Martres, M. J., Michard, R., \& Soru-Iscovici 1966, Ann. Astrophys., 29, 249

Pariat, E., Aulanier, G., Schmieder, B., et al. 2004, ApJ, 614, 1099

Poland, A. I., \& Mariska, J. T. 1986, Sol. Phys., 104, 303

Priest, E. R. 1990, Dynamics of Quiescent Prominences, IAU Colloq. 117, LNP, 363,150

Priest, E. R. 1998, in New Perspectives on Solar Prominences, IAU Colloq. 167, ASP Conf. Ser., 150, 453

Rees, D., López Ariste, A., Thatcher, J., \& Semel, M. 2000, A\&A, 355, 759

Régnier, S., \& Amari, T. 2004, A\&A, 425, 345

Rompolt, B. 1990, Hvar Obs. Bull., 14, 37

Schmieder, B., Aulanier, G., \& López Ariste, A. 2005, in Proc. of the Conf. Chromospheric and Coronal Magnetic fields, ESA SP, 596

Schmieder, B., Raadu, M. A., \& Wiik, J. E. 1991, A\&A, 252, 353

Semel, M., Donati, J.-F., \& Rees, D. E. 1993, A\&A, 278, 231

Socas-Navarro, H., López Ariste, A., \& Lites, B. 2001, ApJ, 553, 949

Titov, V. S., Priest, E. R., \& Demoulin, P. 1993, A\&A, 276, 564

van Ballegooijen, A. A. 2004, ApJ, 612, 519

van Ballegooijen, A. A., \& Martens, P. C. H. 1989, ApJ, 343, 971

Wang, Y.-M. 2001, ApJ, 560, 456

Wu, S. T., Bao, J. J., An, C. H., \& Tandberg-Hanssen, E. 1990, Sol. Phys., 125, 277

Zirker, J. B., Engvold, O., \& Martin, S. F. 1998, Nature, 396, 440

Zong, W. G., Tang, Y. H., Fang, C., et al. 2003, A\&A, 412, 267 